\title{
On Critical Exponents and the Renormalization of the Coupling Constant in Growth Models with Surface Diffusion
}

\author{
H.K. JANSSEN \\ Institut für Theoretische Physik III, Heinrich-Heine-Universität, \\ 40225 Düsseldorf, Germany
}

(August 14, 2018)

\begin{abstract}
It is shown by the method of renormalized field theory that in contrast to a statement based on a mathematically ill-defined invariance transformation and found in most of the recent publications on growth models with surface diffusion, the coupling constant of these models renormalizes nontrivially. This implies that the widely accepted supposedly exact scaling exponents are to be corrected. A two-loop calculation shows that the corrections are small and these exponents seem to be very good approximations.
\end{abstract}

PACS-numbers: 64.60.Ak, 64.60.Ht, 05.40.+j, 68.35.Rh

There has been great interest in nonequilibrium growth processes of surfaces and interfaces in the last ten years (for general overviews on the work in this area of statistical physics and the related literature see the articles of Halpin-Healy and Zhang [1], Krug [2, 3] and the book of Barabasy and Stanley [4]). Recently deposition processes in which surface diffusion constitutes the dominant relaxation mechanism have been intensively studied. Besides the simulational work on many different models, stochastic differential equations for the surface growth with surface diffusion are proposed and studied by scaling and one-loop momentum-shell renormalization methods in order to find out the universal scaling behavior. An apparently mathematically ill-defined invariance transformation leading to supposedly exact scaling exponents plays a crucial role in this analytical work. This transformation and its consequences have been taken over, apparently without careful examination, in most of the related publications and overviews [1, [1], and are now widely accepted in the community of surface-growth physicists.

In this letter I explicitly show that the widely accepted invariance is indeed wrong and the supposedly exact exponents are to be corrected. I will use the systematic method of renormalized field theory (see e.g. [5] 8 ) and calculate the corrections in an $\varepsilon$-expansion to second order.

I consider a system evolving by diffusion of atoms at a surface of (suitably scaled) height $s(\mathbf{x}, t)$ over a $d$ dimensional hyper-plane. Thus the deterministic part of the equation of motion for the stochastic dynamical variable $s$ has to be of the conserved form

$$
\begin{aligned}
\lambda^{-1} \frac{\partial s}{\partial t} & =-\nabla^{2}\left[\tau s+\nabla^{2} s+\frac{g}{2}(\nabla s)^{2}\right]+\zeta \\
\left\langle\zeta(\mathbf{x}, t) \zeta\left(\mathbf{x}^{\prime}, t^{\prime}\right)\right\rangle & =2 \lambda^{-1}\left(-\nabla^{2}\right)^{a} \delta\left(\mathbf{x}-\mathbf{x}^{\prime}\right) \delta\left(t-t^{\prime}\right) .
\end{aligned}
$$

Here the parameter $a=1$ in the fully conserved case originally studied by Sun, Guo, and Grant [] in their first exploration of non-KPZ universality classes (with $\tau=0$ ). If the noise is not conserved (shot noise) the parameter $a=0$. This case of (11) was introduced in the first papers on ideal molecular beam epitaxy (MBE) 10 12]. In the Langevin equation (11) $\lambda$ is a kinetic coefficient and $g$ is the coupling constant. The behavior near the critical point $\tau=0$ is of primary interest. For $\tau>0$ the equations (11) are eventually driven by renormalization to the linear Edwards-Wilkinson equations both with nonconserved or conserved noise.

For a generalization of the Galilean invariance (tilt symmetry) of the KPZ equation [13 Sun, Guo, and Grant introduced as a symmetry of (1) the coordinate transformation

$$
s \rightarrow s+\mathbf{a} \cdot \mathbf{x}, \quad \mathbf{x} \rightarrow \mathbf{x}-\lambda g t \mathbf{a} \nabla^{2}
$$

which appears to be mathematically ill-defined because it mixes coordinates and linear differential operators in a coordinate substitution rule (the questionableness of the rule (2) shows up in a definition of the transformation of a product of functions $\left.f_{1}(\mathbf{x}) f_{2}(\mathbf{x})=f_{2}(\mathbf{x}) f_{1}(\mathbf{x})\right)$. Based on the transformation (2) the authors state that the coupling constant $g$ changes only trivially under renormalization and derive supposedly exact scaling exponents. The ill-defined transformation (2) has been used for various equations of the type (11) to derive critical exponents. Only few authors express some doubt concerning the use of (2) 11, 14.

In the following I show by an explicit two-loop calculation that the coupling constant does indeed change non-trivially under renormalization. Therefore the supposedly exact exponents are to be corrected by a quantity $\delta:$

$$
\chi=\frac{\varepsilon}{3}-\delta, \quad z=4-\frac{\varepsilon}{3}-2 \delta
$$

where $\varepsilon=d_{c}-d$ is the deviation from the upper critical dimension $d_{c}$. The roughening exponent $\chi$ and the dynamic exponent $z$ are defined by the structure function $\left\langle|s(\mathbf{x}, t)-s(\mathbf{0}, t)|^{2}\right\rangle \sim|\mathbf{x}|^{2 \chi} f\left(t /|\mathbf{x}|^{z}\right)$, where $f$ is a scaling function. I calculate the correction $\delta$ to order $\varepsilon^{2}$ 
and find that it is always a small but nonzero positive quantity which has not been detected in simulations up to now.

To develop a renormalized field theory I introduce the dynamic functional [15] 18,7] corresponding to the Langevin equations (1)

$$
\begin{aligned}
\mathcal{J}= & \int d^{d} x d t \lambda\left\{\lambda^{-1} \widetilde{s} \dot{s}+\tau \nabla \widetilde{s} \nabla s\right. \\
& \left.+\nabla^{2} \widetilde{s}\left[\nabla^{2} s+\frac{g}{2}(\nabla s)^{2}\right]-\left(\nabla^{a} \widetilde{s}\right)^{2}\right\} .
\end{aligned}
$$

Here $\widetilde{s}$ denotes the response field (the MSR [19] auxiliary variable). Now all response and correlation functions can be calculated by path integrals with the weight $\exp (-\mathcal{J}[\widetilde{s}, s])$. The expansion of the path integrals about the Gaussian part of $\mathcal{J}$ generates the (diagrammatic) perturbation theory which has to be regularized and renormalized because the series are UV-divergent at the upper critical dimensions that are given by $d_{c}=4$ for $a=0$ and $d_{c}=2$ for $a=1$. The propagator $G(\mathbf{q}, t)=\theta(t) \exp (-\kappa(q) t)$ with $\kappa(q)=\lambda q^{2}\left(\tau+q^{2}\right)$ and the correlator $C(\mathbf{q}, t)=\chi(q) \exp (-\kappa(q)|t|)$ with $\chi(q)=q^{2(a-1)}\left(\tau+q^{2}\right)^{-1}$ are considered as functions of the momentum and time. The step function $\theta(t)$ is defined with $\theta(0)=0$. The vertex is given by $V\left(\mathbf{q}_{1} ; \mathbf{q}_{2}, \mathbf{q}_{3}\right)=\mathbf{q}_{2} \cdot \mathbf{q}_{3} v\left(q_{1}\right)$ with $v(q)=-\lambda g q^{2}$. Here $\mathbf{q}_{2}, \mathbf{q}_{3}$ are the ingoing momenta of the two $s$-legs and $\mathbf{q}_{1}=\mathbf{q}_{2}+\mathbf{q}_{3}$ denotes the outgoing momentum of the $\widetilde{s}$-leg. Thus the diagrammatic expansions lead only to one-particle irreducible vertex functions with (at least) a factor $q^{2}$ at each (amputated) $\widetilde{s}$-leg and a factor $\mathbf{q}$ at each $s$-leg. A look at the naïve dimensions of these vertex functions shows that only primitive divergencies arise which correspond to the terms $\nabla^{2} \widetilde{s} \nabla^{2} s$ and $\nabla^{2} \widetilde{s}(\nabla s)^{2}$ in the dynamic functional (4). Therefore the model is renormalizable by the scheme

$$
\begin{aligned}
& s \rightarrow \stackrel{\circ}{s}=Z^{1 / 2} s, \quad \widetilde{s} \rightarrow \stackrel{\circ}{s}=Z^{-1 / 2} \widetilde{s}, \quad \lambda \rightarrow \stackrel{\circ}{\lambda}=Z \lambda \\
& \tau \rightarrow \stackrel{\circ}{\tau}=Z^{-1} \tau, \quad g^{2} \rightarrow \stackrel{\circ}{g}^{2}=A_{\varepsilon} \mu^{\varepsilon} Z^{-3} Z_{u} u .
\end{aligned}
$$

$A_{\varepsilon}=(4 \pi)^{d / 2} \Gamma(1+\varepsilon / 2)^{-1}$ is a suitable constant and $\mu$ is the usual inverse external length scale which makes the renormalized coupling constant $u$ dimensionless. The two renormalization constants $Z$ and $Z_{u}$ are determined by minimal renormalization. One easily gets (corresponding to the known 1-loop results)

$$
Z=1-2^{a-2} \frac{u}{\varepsilon}+O\left(u^{2}\right), \quad Z_{u}=1+O\left(u^{2}\right) .
$$

The incorrect assertion based on the invariance transformation (2) is that $Z_{u}=1$ to all orders of $u$. In the following I give the derivation of the second order contribution.

The calculation is greatly simplified due to the following properties of the diagrammatic contributions. Consider a diagram in the momentum-time picture. In this representation the restrictions which causality imposes are more easily exploited than in the momentumfrequency picture; note in particular the $\theta$-function of the propagator. Due to causality the vertices are ordered from right to left with increasing time labels. Thus, a diagram with $n$ vertices consists of $(n-1)$ time segments. A Fourier transformation from time differences to frequencies then generates a dynamical factor

$$
D(\{\mathbf{q}, \omega\})=\left[i \sum_{\alpha} \omega_{\alpha}+\sum_{k} \kappa\left(\mathbf{q}_{k}\right)\right]^{-1}
$$

for each time segment. Here $\sum_{\alpha} \omega_{\alpha}$ is the sum over all external frequencies flowing into the segment from the right, and the set $\left\{\mathbf{q}_{k}\right\}$ are the internal momenta running from right to left within the segment. In addition to the dynamical factors, each correlator contributes a factor $\chi(\mathbf{q})$ and each vertex a factor $V\left(\mathbf{q}_{1} ; \mathbf{q}_{2}, \mathbf{q}_{3}\right)$. Finally one has to integrate over the internal momenta.

I now consider one-particle irreducible diagrams with amputated legs as contributions to the vertex functions. For the determination of the UV-divergencies all external momenta and frequencies can be set to zero if the external momenta coming from the derivatives at the external legs are included in the amputation procedure. To find the renormalization factor $Z_{u}$, one needs the vertex function which results from the diagrams with amputated legs corresponding to one $\nabla^{2} \widetilde{s}$ and two $(\nabla s)$. I now discuss how certain diagrams cancel (this discussion is analogous to that in 20]). Consider the earliest vertex of the diagram. If this vertex has an amputated $(\nabla s)$-leg, its contributions coming from different permutations of the propagator (Fig. 1a) add to zero because $\mathbf{q} v(-q) \chi(q)-\mathbf{q} v(q) \chi(-q)=0$. It is essential for this cancellation that the vertex considered carries the earliest time argument, since otherwise the straightforward permutation of propagators is prohibited by causality. Especially, by this cancellation all one-loop vertex functions with zero external momenta vanish, and it follows that the coupling renormalizes only trivially to this order. If the earliest vertex is an internal one, the contributions coming from the propagator permutations are added up to the summed vertex (Fig. 2a)

$$
\begin{aligned}
S\left(\mathbf{q}_{1}, \mathbf{q}_{2}, \mathbf{q}_{3}\right)= & \frac{V\left(\mathbf{q}_{1} ; \mathbf{q}_{2}, \mathbf{q}_{3}\right)}{\chi\left(q_{1}\right)} \\
& +\frac{V\left(\mathbf{q}_{2} ; \mathbf{q}_{3}, \mathbf{q}_{1}\right)}{\chi\left(q_{2}\right)}+\frac{V\left(\mathbf{q}_{3} ; \mathbf{q}_{1}, \mathbf{q}_{2}\right)}{\chi\left(q_{3}\right)}
\end{aligned}
$$

with $\mathbf{q}_{1}+\mathbf{q}_{2}+\mathbf{q}_{3}=0$. The factors $\chi^{-1}$ result from the exchange of a propagator line with the corresponding correlator line.

If in the diagrams under consideration the two vertices with the amputated $(\nabla s)$-legs are deleted, all possible diagrams with internal earliest vertex (the only ones that 
are nonzero) reduce to one kernel-diagram (Fig. 2b) with a contribution

$$
\begin{aligned}
K\left(\mathbf{q}_{1}, \mathbf{q}_{2}, \mathbf{q}_{3}\right)= & \frac{\lambda g q_{1}^{2}}{2} \frac{V\left(\mathbf{q}_{1} ; \mathbf{q}_{2}, \mathbf{q}_{3}\right)}{2 \kappa\left(q_{1}\right)} \\
& \cdot \frac{\chi\left(q_{1}\right) \chi\left(q_{2}\right) \chi\left(q_{3}\right)}{\kappa\left(q_{1}\right)+\kappa\left(q_{2}\right)+\kappa\left(q_{3}\right)} S\left(\mathbf{q}_{1}, \mathbf{q}_{2}, \mathbf{q}_{3}\right)
\end{aligned}
$$

which is self-explaining (the first factor $1 / 2$ results from the double connection of the two internal vertices). Inserting back the external vertices allows the discussion of a second cancellation mechanism. The insertions of an amputated vertex in a time segment with only two internal lines add to zero if all external momenta are zero (Fig. 1b) because $\mathbf{q} v(q)-\mathbf{q} v(-q)=0$. Therefore only the insertions into the second time segment with three internal lines of the kernel-diagram (Fig. 2b) may lead to a nonvanishing contribution. The result of the addition of the insertions of one amputated external vertex with zero external momentum into a three-line segment (Fig. 1c) is the factor

$$
\mathbf{W}\left(\mathbf{q}_{1}, \mathbf{q}_{2}, \mathbf{q}_{3}\right)=\frac{\mathbf{q}_{1} v\left(q_{1}\right)+\mathbf{q}_{2} v\left(q_{2}\right)+\mathbf{q}_{3} v\left(q_{3}\right)}{\kappa\left(q_{1}\right)+\kappa\left(q_{2}\right)+\kappa\left(q_{3}\right)}
$$

where $\mathbf{q}_{1}+\mathbf{q}_{2}+\mathbf{q}_{3}=0$, and the denominator arises because a new time segment is produced by an insertion. In the case of the KPZ equation the functions $v\left(q_{i}\right)$ are constants and it follows that these insertions add to zero: $\mathbf{W}=0$, a result which is easily generalized to insertions in all segments and has its origin in the Galilean invariance [20]. But in the models considered here $v(q) \sim q^{2}$, and it is easily seen that $\mathbf{W}\left(\mathbf{q}_{1}, \mathbf{q}_{2}, \mathbf{q}_{3}\right)=0$ only in the case of at least one vanishing momentum $\mathbf{q}_{i}$. In general $\mathbf{W} \neq 0$ which eventually disproves all statements on the trivial renormalization of the coupling constant. The insertions of the two external vertices then lead to the tensor $\mathbf{W} \otimes \mathbf{W}$. But after integration over the momenta the integral has to be a rotational invariant. Thus it must be proportional to the $d$-dimensional unit tensor and its prefactor can be determined by taking the trace. Therefore instead of the integral with the tensor $\mathbf{W} \otimes \mathbf{W}$ only the integral with the trace $\frac{1}{d} \mathbf{W} \cdot \mathbf{W}$ must be calculated to find the result of the insertion of the two external vertices into the kernel-diagram. Eventually as the contribution of all two loop diagrams to the amputated vertex function with zero external momenta and frequencies I obtain the integrand

$$
I\left(\mathbf{q}_{1}, \mathbf{q}_{2}, \mathbf{q}_{3}\right)=\frac{2}{d} K\left(\mathbf{q}_{1}, \mathbf{q}_{2}, \mathbf{q}_{3}\right) \mathbf{W}\left(\mathbf{q}_{1}, \mathbf{q}_{2}, \mathbf{q}_{3}\right)^{2}
$$

with $\mathbf{q}_{1}+\mathbf{q}_{2}+\mathbf{q}_{3}=0$. The factor 2 arises from the permutation of the external amputated $s$-legs.

Performing the integral over the internal loop momenta $R=(2 \pi)^{-2 d} \int \prod d^{d} q_{i} \delta\left(\mathbf{q}_{1}+\mathbf{q}_{2}+\mathbf{q}_{3}\right) I\left(\mathbf{q}_{1}, \mathbf{q}_{2}, \mathbf{q}_{3}\right)$ one observes that the IR-regulator $\tau>0$ can be neglected in all terms with exception of the dynamical factor $\left(\kappa\left(q_{1}\right)+\kappa\left(q_{2}\right)+\kappa\left(q_{3}\right)\right)^{-3}$. Then the integration over the variable $s=q_{1}^{2}+q_{2}^{2}+q_{3}^{2}$ extracts the $\varepsilon$-pole, and in the remaining part of the integral the dimension $d$ can be safely set to $d_{c}$. Up to trivial angle integrations the result $R$ reduces to a finite two-dimensional integral without any singularities in the integrand. The elimination of the $\varepsilon$-pole from the renormalized vertex function finally leads to a definitely nontrivial renormalization constant

$$
\begin{aligned}
Z_{u} & =1-\frac{B_{a}}{\varepsilon} u^{2}+O\left(u^{3}\right) \\
B_{a} & =\left\{\begin{array}{lll}
0.0057406 & \text { if } & a=0 \\
0.0167998 & \text { if } & a=1
\end{array} .\right.
\end{aligned}
$$

The RG functions are defined by $\gamma=(\partial \ln Z / \partial \ln \mu)_{0}$, $\gamma_{u}=\left(\partial \ln Z_{u} / \partial \ln \mu\right)_{0}$, and $\beta=\mu(\partial u / \partial \mu)_{0}$ (the index 0 denotes differentiation at fixed bare parameters). From the renormalization of the coupling constant (5) one gets $\beta=\left(-\varepsilon+3 \gamma-\gamma_{u}\right) u$. At the stable nontrivial fixed point $u_{*} \neq 0$ with $\beta\left(u_{*}\right)=0$ (for $\varepsilon>0$ ), this equation yields the anomalous scaling dimension $\eta=\gamma\left(u_{*}\right)=\varepsilon / 3+2 \delta$ of the height-field $s$ where I have defined $\delta=\gamma_{u}\left(u_{*}\right) / 6$. From the calculated renormalization factors $(6)$ and (12), the $\gamma$-functions are derived as $\gamma=2^{a-2} u+O\left(u^{2}\right)$ and $\gamma_{u}=2 B_{a} u^{2}+O\left(u^{3}\right)$. Thus from $\beta\left(u_{*}\right)=0$ one finds to first order in the $\varepsilon$-expansion $u_{*}=2^{2-a} \varepsilon / 3+O\left(\varepsilon^{2}\right)$. Hence the correction is given by

$$
\begin{aligned}
& \delta=\frac{4^{2-a}}{27} B_{a} \varepsilon^{2}+O\left(\varepsilon^{3}\right) \\
& =\left\{\begin{array}{ll}
0.01361(\varepsilon / 2)^{2} & \text { if } \quad a=0 \\
0.002489 \varepsilon^{2} & \text { if } \quad a=1
\end{array}+O\left(\varepsilon^{3}\right) .\right.
\end{aligned}
$$

For the physical case of a two-dimensional surface with nonconserved noise $\left(a=0, d_{c}=4, \varepsilon=2\right)$ or a onedimensional surface with conserved noise $\left(a=1, d_{c}=2\right.$, $\varepsilon=1$ ) the numerical prefactors represent the results for $\delta$. As I have stated above, these corrections are always small but nonzero positive numbers. From the anomalous dimension one finds the roughening exponent $\chi=(\varepsilon-\eta) / 2$ and the dynamic exponent $z=4-\eta$ as anticipated in (3). Of course the scaling law $z-2 \chi=4-\varepsilon$ holds, being an exact consequence of the trivial renormalization of the noise. But the relation $4=z+\chi=$ $4+(\varepsilon-3 \eta) / 2=4-3 \delta$, where the first equality comes from the ill-defined symmetry (2), is definitely wrong.

In summary I have shown that in contrast to a statement found in most of the recent publications on growth models with surface diffusion, the coupling constant of these models renormalizes non-trivially. This implies that the widely accepted scaling exponents are to be corrected. But my two-loop result also shows that these corrections are very small as long as one accepts the numerical result at least as a qualitative one with respect to the order of magnitude. Thus the supposedly exact exponents seem to be a very good approximation and the deviations are not seen in simulations and experiments up to 
now. I suppose that the origin of the smallness of the correction is found in the gradient coupling leading to many (but incomplete) cancellations between diagrams as well as within the internal momentum integrals. Therefore I expect the mode-coupling approach which neglects vertex corrections completely to be appropriate here. I will come back to this in the future.

I remark that the calculation presented in this letter is the first two-loop calculation for the vertex functions of the stochastic models considered here. Concerning these models the work of Das Sarma and Kotlyar 21] contains a collection of two-loop self-energy diagrams. However these diagrams are irrelevant for the problem of the second-order renormalization of the coupling constant. Thus the authors cite the one-loop result $\chi+z=4$ for the model with nonconserved noise. Based on the illdefined transformation (2) they believe this scaling law to hold to all orders in perturbation theory. As I have shown this statement is incorrect.

\section{ACKNOWLEDGMENTS}

I thank S. Theiss for a critical reading of the manuscript. This work has been supported in part by the SFB 237 of the Deutsche Forschungsgemeinschaft.

[1] T. Halpin-Healy and Y.-C. Zhang, Phys. Rep. 254, 215 (1995).

[2] J. Krug, in Scale Invariance, Interfaces, and NonEquilibrium Dynamics, edited by A. McKane, M. Droz, J. Vannimenus, and D. Wolf (Plenum, New York, 1995).

[3] J. Krug, to appear in Adv. of Phys.

[4] A.-L. Barabási and H. E. Stanley, Fractal Concepts in Surface Growth, (University Press, Cambridge, 1995).

[5] D. J. Amit, Field Theory, the Renormalization Group and Critical Phenomena, (World Scientific, Singapore, 1984).
[6] J. Zinn-Justin, Quantum Field Theory and Critical Phenomena, 2nd revised edition, (Clarendon, Oxford, 1993).

[7] R. Bausch, H. K. Janssen, and H. Wagner, Z. Phys. B 24, 113 (1976).

[8] C. DeDominicis and L. Peliti, Phys. Rev. B 18, 353 (1978).

[9] T. Sun, H. Guo, and M. Grant, Phys. Rev. A 40, 6763 (1989).

[10] D. E. Wolf and J. Villain, Europhys. Lett. 13, 389 (1990).

[11] J. Villain, J. Phys. I (France) 1, 19 (1991).

[12] Z.-W. Lai and S. Das Sarma, Phys. Rev. Lett. 66, 2348 (1991).

[13] M. Kardar, G. Parisi, and Y.-C. Zhang, Phys. Rev. Lett. 56, 889 (1986).

[14] L.-H. Tang and T. Nattermann, Phys. Rev. Lett. 66, 2889 (1991).

[15] C. DeDominicis, J. Phys. (France) Colloq. 37, C247 (1976).

[16] H. K. Janssen, Z. Phys. B 23, 377 (1976).

[17] H. K. Janssen, in Dynamical Critical Phenomena and Related Topics (Lecture Notes in Physics, Vol. 104), edited by C. P. Enz, (Springer, Heidelberg, 1989).

[18] H. K. Janssen, in From Phase Transitions to Chaos, edited by G. Györgyi, I. Kondor, L. Sasvári, and T. Tél, (Worl Scientific, Singapore, 1992).

[19] P. C. Martin, E. D. Siggia, and H. H. Rose, Phys. Rev. A 8, 423 (1973).

[20] H. K. Janssen and B. Schmittmann, Z. Phys. B 64, 503 (1986).

[21] S. Das Sarma and R. Kotlyar, Phys. Rev. E 50, R4275 (1994).

FIG. 1. Cancellation of diagrams: a) an earliest external vertex, b) an external vertex inserted in a two-line segment, but c) the insertions of an external vertex in a three-line segment in all possible ways do not cancel in general, (time increases always from right to left, the arrows indicate the time direction of the propagators).

FIG. 2. The fundamental diagram: a) an internal earliest vertex with all its propagator permutations, b) the two-loop kernel diagram, (time and momenta flow from right to left). 
Fig. 1a
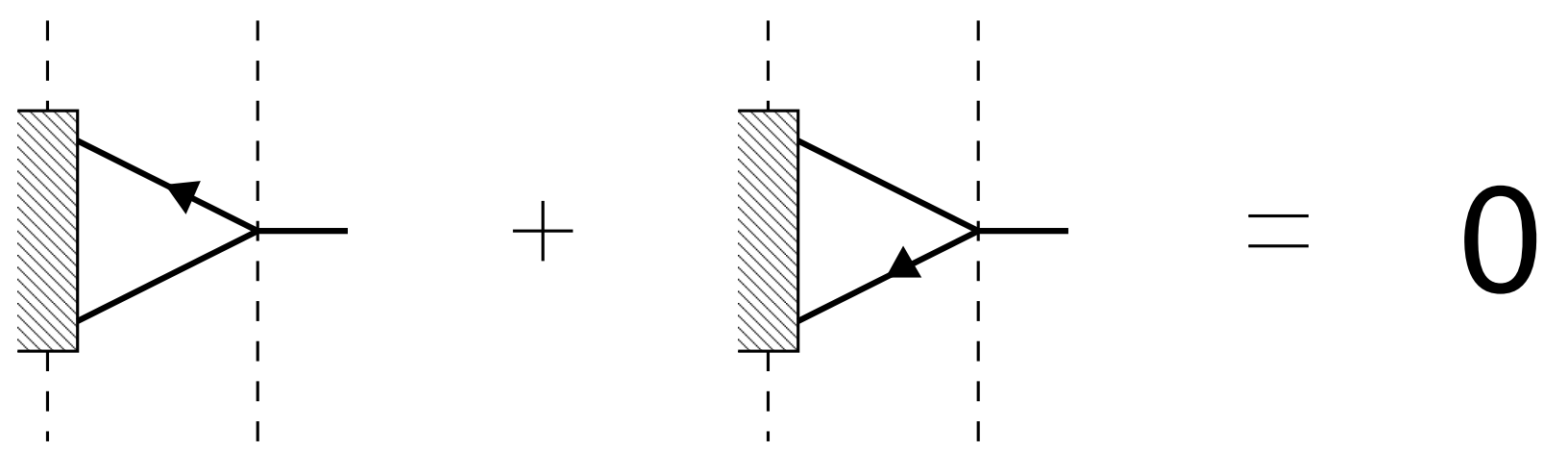

Fig. 1b
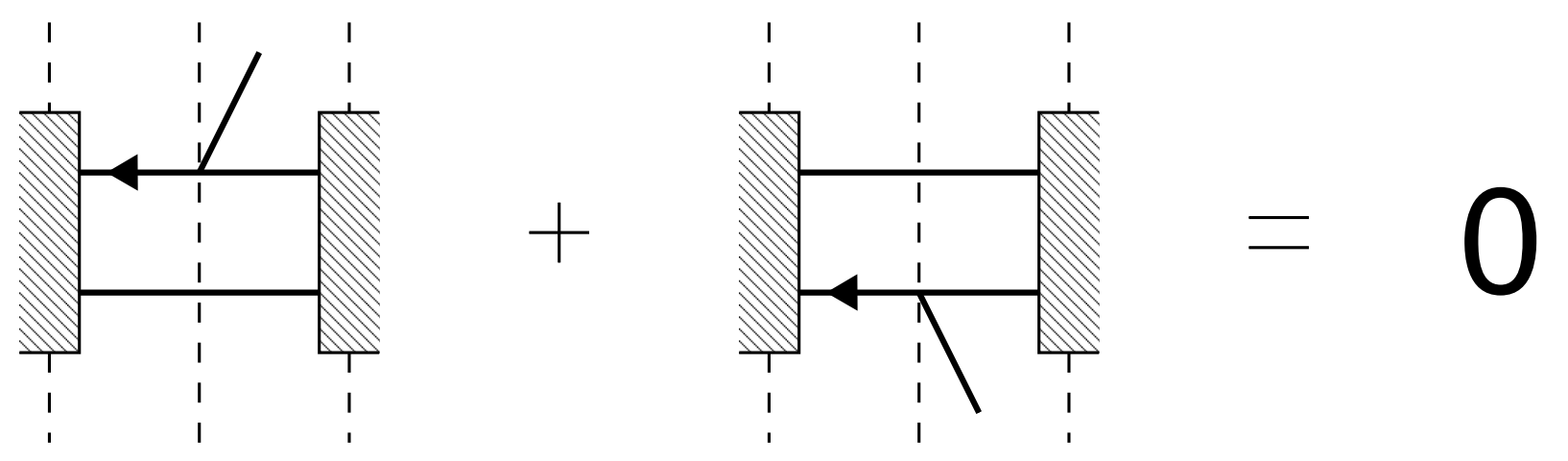

Fig. 1c
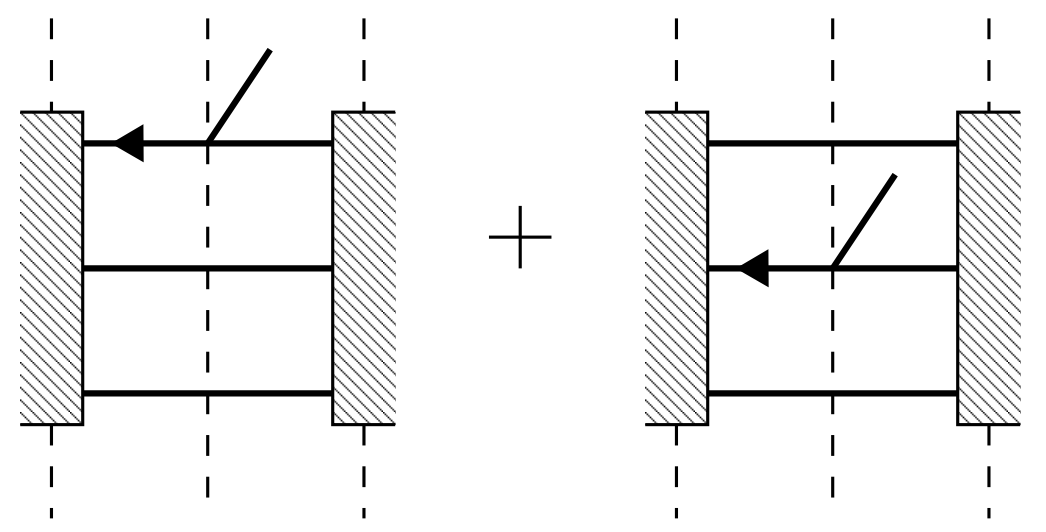

$\neq 0$ 
Fig. 2a

$\eta=\rangle+\gg+\lambda$

Fig. 2b

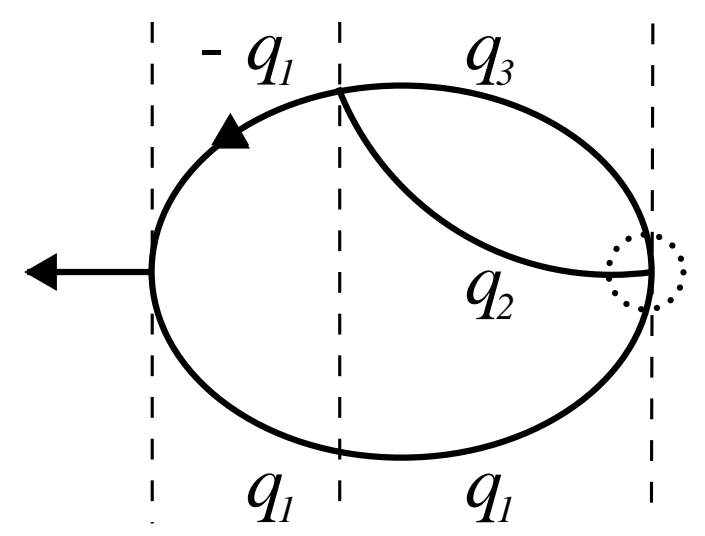

